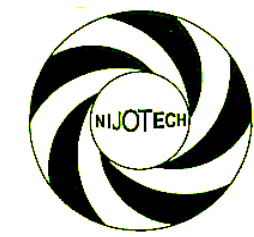

Nigerian Journal of Technology (NIJOTECH)

Vol. 38, No. 2, April 2019, pp. 406 - 415

Copyright@ Faculty of Engineering, University of Nigeria, Nsukka,

Print ISSN: 0331-8443, Electronic ISSN: 2467-8821

www.nijotech.com

http://dx.doi.org/10.4314/njt.v38i2.17

\title{
DESIGN OF A GPS-BASED ACADEMIC PERSONNEL CLOCKING SYSTEM
}

\author{
T. C. Adeniran ${ }^{1,}{ }^{*}$, A. O. Anyaegbu ${ }^{2}$, L. A. Olawoyin ${ }^{3}$ and A. O. Ajagbe ${ }^{4}$ \\ $\mathbf{1}, \mathbf{2}, \mathbf{3}, \mathbf{4}$, DEPT OF TELECOMMUNICATION SCIENCE, UNIVERSITY OF ILORIN, ILORIN, KWARA STATE. NIGERIA. \\ E-mail addresses: ${ }^{1}$ adeniran.tc@unilorin.edu.ng, 2 alfredanyaegbu@gmail.com, \\ 3 lolawoyin@gmail.com, ${ }^{4}$ talk2jibola247@yahoo.com
}

\begin{abstract}
Ensuring optimal productivity in the workplace is a major concern for employers of labour; hence the proposition of clocking solutions for employees in a bid to track their presence and punctuality at their duty posts. However, the peculiarity of the academic environment makes general methods of clocking unsuitable for lecturers. This paper presents design and implementation of a GPS-based clocking solution for academic personnel using a web App and an Android client. The web App; written in NodeJS and hosted on Heroku, in conjunction with the database (Mongo DB), registers and holds the schedule details of the lecturers. The android client which is installed on lecturers' mobiles, works in synergy with the web App to accomplish clocking. This concept is reliant on GPS, thus, the lecturers' mobiles require a clear view of the sky for successful clocking. The aspect of security can be improved upon with a few tweaks in the applications' program codes. In all, the system utilizes existing and readily available resources to achieve clocking of academic personnel.
\end{abstract}

Keywords: Global Positioning System (GPS), Telecommunication Science (TCS), Java, MongoDB, NavSTAR, NodeJS and clocking.

\section{INTRODUCTION}

Clocking is a method used by employers of labour to track dutifulness of employees. It has evolved over the years from manual to tech-based solutions. Options include biometric, cards and tags, location-based solutions. Global Positioning System (GPS) is a spacebased satellite system that provides reliable time and location information irrespective of weather condition on or near the earth surface, if, there is an unobstructed line of sight to four or more GPS satellites [1, 2]. Thus, it makes a whole lot of sense to capitalise on the enormous availability, reliability and coverage offered by such a system. GPS-based clocking is a location-based clocking solution that employs the GPS network's Standard Positioning Services to ascertain the location of employees at specific times, thus tracking dutifulness. The academic environment is a peculiar one and is therefore different from the typical workplace or industrial environment. In an industrial setting, a lack of dutifulness on the part of members of staff will result in loss of working hours, which in turn translates to reduced productivity (in terms of quality and quantity of products) and hence decreased profits for the organisation. However, the effects of noncompliance to schedule and lack of dutifulness in the academic community are more far-reaching and often disastrous $[3,4]$. Some of these include but not limited to, reduction in the overall quality of the academic experience, subpar products coming off the academic production line, a shortage of quality workforce to drive critical sectors of the economy, a decline in national GDP, etcetera. Thus, the research activities on implementation of a cost-effective method of clocking for this peculiar workforce to maintain the integrity and ensure continuity and consistency are receiving serious attention in order to discourage absenteeism and negligence of duty [3]. A typical example of this is the one carried out by [3]; where the overall effect of the implementation of a time and attendance system at Stellenbosch municipality is examined using questionnaire method.

It is asserted in [3] that the unreliability and ineffectiveness of manual clocking of employees

* Corresponding author, tel: + 234-816- $385-7493$ 
necessitated the recommendation of an automated system of employee clocking.

In order to solve problems associated with manual clocking mechanisms, [5] designed an RF-based clocking and access control system for office use. The system comprises three modules: the central station module, the user module and the grant access module [5]. Every employee has his or her user unit. The employee, in the event of losing such unit, can contact the central station via a mobile phone or using an emergency unit at the central station. The system functions by utilising radio transmitters and receivers incorporated into the various modules to enable clockins and clock-outs and also grant entry and access to resources within the workplace. However, the system proposed by [5] is expensive.

In [6], a system of attendance incorporating automated access control using RFID technology is proposed. The system is a combination of hardware and software. The hardware comprised of the readers and tags, otherwise known as the interrogators and labels respectively. As per the tags, there are active tags; which are battery-powered, passive tags; which do not possess a battery and battery-assisted passive tags; which possess a small battery and unlike the outright active tag, and it is activated only when in the presence of an RFID reader [6]. Access is granted to authorised cardholders while his/her attendance is simultaneously taken. The system proposed by [6] is an improvement on manual clocking or attendance, but still prone to issues with buddy punching. Again, implementation and deployment for a reasonably sized organisation will incur significant costs. Loss or theft of RFID tags/cards is also a problem and a security loophole [6][7].

In [8], a system is proposed for employee clocking, that relies on the location of smart gadgets such as mobile phones and wearable devices. This system boycotts most of the infrastructure costs and hassles of having magnetic stripe cards, RFID card, barcode badges and biometric equipment. The method exploits the enormous potentials of location technologies such as GPS, cellular, Wi-Fi, Bluetooth etcetera.

From the preceding, it is evident that several attempts and efforts have been directed at automating management of employees in the workplace to increase productivity and reduce unjustified labour costs.

In today's world, apart from clocking, there isneed to keep tracking the movement and position of person(s) or object(s). in order to achieve these, [9] identifies the usage of Geo-locational technologies such as Beacon and dead reckoning systems for location of position of an object in motion. are initial tracking concepts that are most relevant when the operational area is small and the route taken by the tracked object is static [9]. Other techniques are LORAN and GLONASS.

\section{MATERIALS AND METHODS}

\subsection{GPS Architecture}

The GPS is a system developed for, funded by and operated by the United States Department of Defense $[1,10,11]$. It was primarily meant for military use but has found the widespread civilian application. The GPS is a network of 24 satellites or space vehicles (nominally, because new satellites are launched continually to take over from older ones which are at the end of their life cycles) arranged in six different orbits [9]. These orbits are 12-hour orbits. The space vehicles transmit radio signals from space.

Fig. 1 shows that GPS is divided into three segments: The space, user and control segments [9]. The space vehicles (SVs) constitute the space segment. The user segment comprises of receivers (which could be installed in vehicles, smart phones or standalone GPS devices) that use the radio signals emitted from the SVs to compute the distance between them and the satellite [9]. According to [9], for a user to compute its location, it needs to lock onto signals from at least three different SVs - a concept more accurately known as trilateration. The arrangement of the GPS constellation is such that at any point on the earth surface, at least five SVs are in view, and utilising this, a three-dimensional position (longitude, latitude and altitude) of the user is determined [11].

The control segment of the GPS network is made up of five monitor earth stations with four ground antennas in different locations around the world [9, 11]. The main control earth station is located at the Schriever Air Force Base in Colorado USA. All the information from the other control stations is relayed to this master station which is saddled with the responsibility of coordinating the overall system plus uploading of correction data to the SVs [11, 12].

According to [12], the GPS is also used for time and frequency dissemination. This is because of the very high accuracy of the atomic clocks aboard the SVs. This highly accurate time and frequency system are critical for telecommunication systems, observatories and research laboratories. 


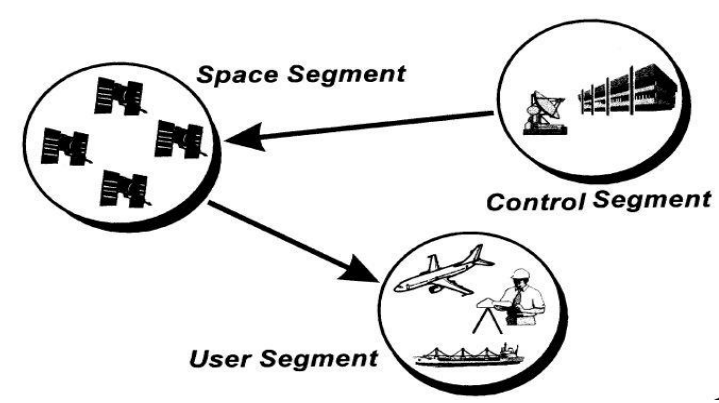

Fig. 1: GPS segments [12]

\subsection{GPS Service Levels}

The U.S Department of defence - owners and operators of the GPS have delineated the positioning services of the GPS system into two levels: The Precise and Standard Positioning Services

The Precise Positioning Service (PPS) offers high-level accuracy positioning service reserved for the U.S military, their allies and select non-military users authorised by the U.S government. This class of users enjoy highly accurate GPS time and position data [2]. For PPS, accuracy is 17.8 meters horizontally, 27.7 meters vertically and 100 nanoseconds for time [9, 10].

The Standard Positioning Service (SPS) is meant for civilian use. It is offered free of charge. The accuracy for SPS is low because it is intentionally degraded by the operators of the GPS [9]. This intentional degradation is referred to as Selective Availability. Accuracy for the standard services is 100 meters horizontally, 150 meters vertically and 167 nanoseconds. However, Selective Availability has been since turned off by the U.S government.

\section{3 GPS Satellites}

The GPS satellites are manufactured by Rockwell International in the USA; and named NAVSTAR [1, 10]. Each satellite spans about 5meters with its solar panels fully extended. Weighing about $900 \mathrm{~kg}$, the satellites have a lifespan of about seven and a half years. There are about six orbital planes in the GPS constellation, and each plane holds about four satellites. Each orbit has an altitude of about 20,200 $\mathrm{km}$, half the altitude of a geostationary satellite orbit. This, therefore, means that the orbits are 12-hour orbits. They are inclined at $55^{\circ}$ to the equatorial plane with each of the orbital planes spaced at $60^{\circ}$ from each other [12].

\subsection{GPS Signals}

The GPS satellites transmit on two different frequencies: L1 and L2 [13]. "The L1 frequency is 1575.42 megahertz and is responsible for carrying the navigation message and the Standard Positioning Services code signal" [9]. For the L2 frequency, it is responsible for carrying the ionospheric delay which is used by the Precise Positioning Services receivers [9]. The L2 frequency is $1227.60 \mathrm{MHz}$ [9].From the perspective of the average user, signaling in the GPS network is a simplex form of communication. This is to say; transmission occurs only in one direction (from the space vehicles to the receivers on the ground). Thus, there is no uplink between the user and the satellites. The only uplink in the system exists between the GPS constellation and the control stations that upload correction data to the satellites. The GPS signals are resistant to jamming and interference [13]. The working of the system requires a receiver to be able to lock on to signals coming from at least four GPS satellites, therefore, a clear view of the sky is necessary for proper functioning [9]. In other words, GPS is not designed for use indoors or in places where the RF signal power is diminished [13]. According to [9], the GPS navigation message is made up of 1500bit data frame which is transmitted every 30 seconds; and this frame is divided into five 300 -bit subframes. The subframes carry clock and orbital data and also system data $[9,11]$. A set of 125 data frames make up a GPS navigation message.

\section{PROPOSED SYSTEM}

\section{1 Implementation of the system}

The block diagram of the proposed system is shown in Fig. 2. The mobile and web applications work in synergy to ensure that academic personnel clock in and out during their allotted lecture time. The clients' application runs on GPS-enabled Android devices, which picks up the user's geolocation details and routes the same to the server which makes a judgment on whether the user is on site or not. The judgment is based on a pre-set configuration which comprises of the longitude and latitude of the designated lecture venue. In conjunction with a location offset (tolerance) range within which an actual attendance can still be registered. The server takes in several inputs in the following fields such as Lecturer's ID, Lecturer's Name, Authorization Code, Day of the Week, Course, Course Code, Venue, Start time, End time, Longitude, Latitude and Tolerance. On the Android application, the user's lecture schedule is displayed right after the login window, after which the clock-in button is visible. 


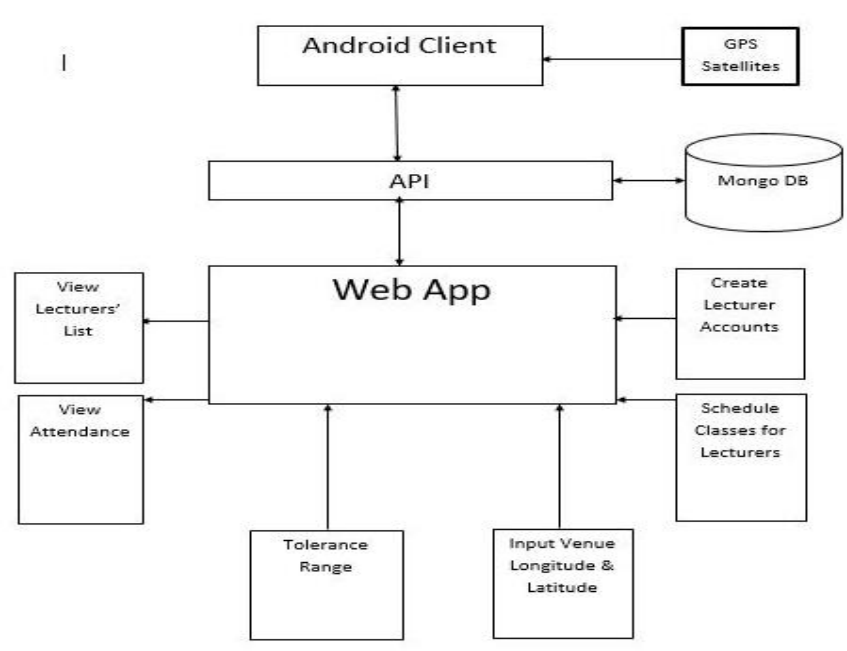

Fig. 2: System Block diagram

\subsection{Software development for the proposed system}

Fig. 4 shows the flow-chart for the implementation of the software of the proposed system. The web application is written in node.js [14]. The choice of node.js is mainly for scalability reasons as this is single-threaded and asynchronous, enabling the web application to be better adapted to handling multiple requests simultaneously. It is, however, important to note that while node.js is not a full-blown programming language, yet it is more than just a framework. The asynchronous nature of node.js ensures that code execution is never blocked as a result of waiting for an operation to go to completion. This is achieved using the call-back functionality [15]. Node.js makes it reasonably straightforward to build server and client applications that communicate with each other using streams and pipes. The choice of this language is because it is suitable for writing real-time web applications that are not CPU-intensive [15]. Other essential features of the language are, being event-driven, asynchronous execution and modularity. However, using node.js for applications that involve heavy computational operations could backfire because the event loop is blocked and the web server's ability to handle other concurrent requests is impacted negatively.

The web application is then linked to a database that functions to hold the details of the registered users (lecturers), the lecture schedules and the attendance records. The database chosen for this clocking system is MongoDB. MongoDB as represented in Fig.3, is a general purpose and open source database that is flexible and scalable $[14,15]$. It is a NoSQL database that shifts away from the traditional relational database management systems (RDBMS). MongoDB groups documents into collections, and the collections into databases. A single instance of MongoDB can host several databases. In MongoDB, 'document' is to 'collection' what 'row' is to 'table' in relational databases. While working with MongoDB, it is good practice to put documents of the same type in the same collection. This makes access easier. We can also have subcollections in the MongoDB hierarchy [15]. The administration is made easier by the MongoDB Shell. Query in MongoDB are performed using the 'find ( $)^{\prime}$ or 'find one ( $)^{\prime}$ functions [15]. It is important to note that MongoDB does not incorporate any built-in encryption or security mechanism [14].MongoDB and NodeJS make a perfect fit as both are well adapted to handling concurrent requests and also are JSON-friendly.

\subsection{System Operation}

The targeted users of the system are the administrator and the lecturers. The administrators man the web application while all the lecturers have the android client installed on their smartphones. The roles of lecturers are mainly to view lecture schedule and clock-in. Other role of all the users are represented in Fig. 4. The system makes attendance decision by first, using the received geo-coordinates of the user mobile to compute the distance between the user mobile and the pre-set geo-coordinates of the designated venue for the lecture under consideration at that point in time.

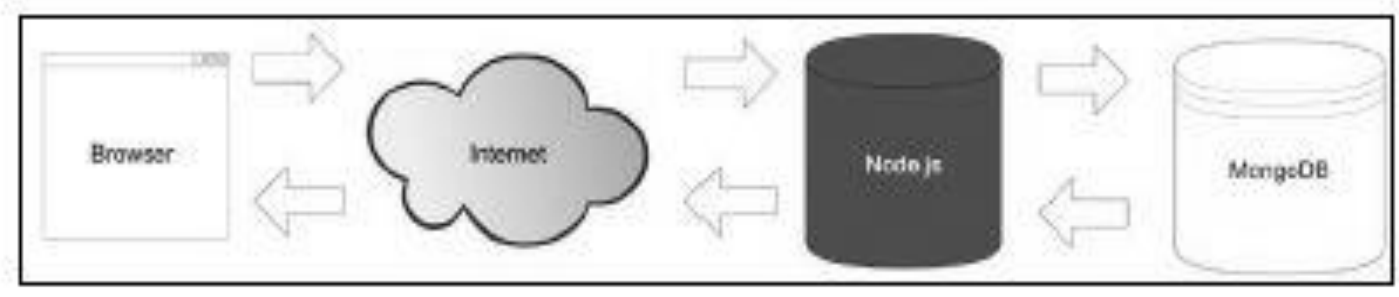

Fig. 3: MongoDB and NodeJS web applications [15] 


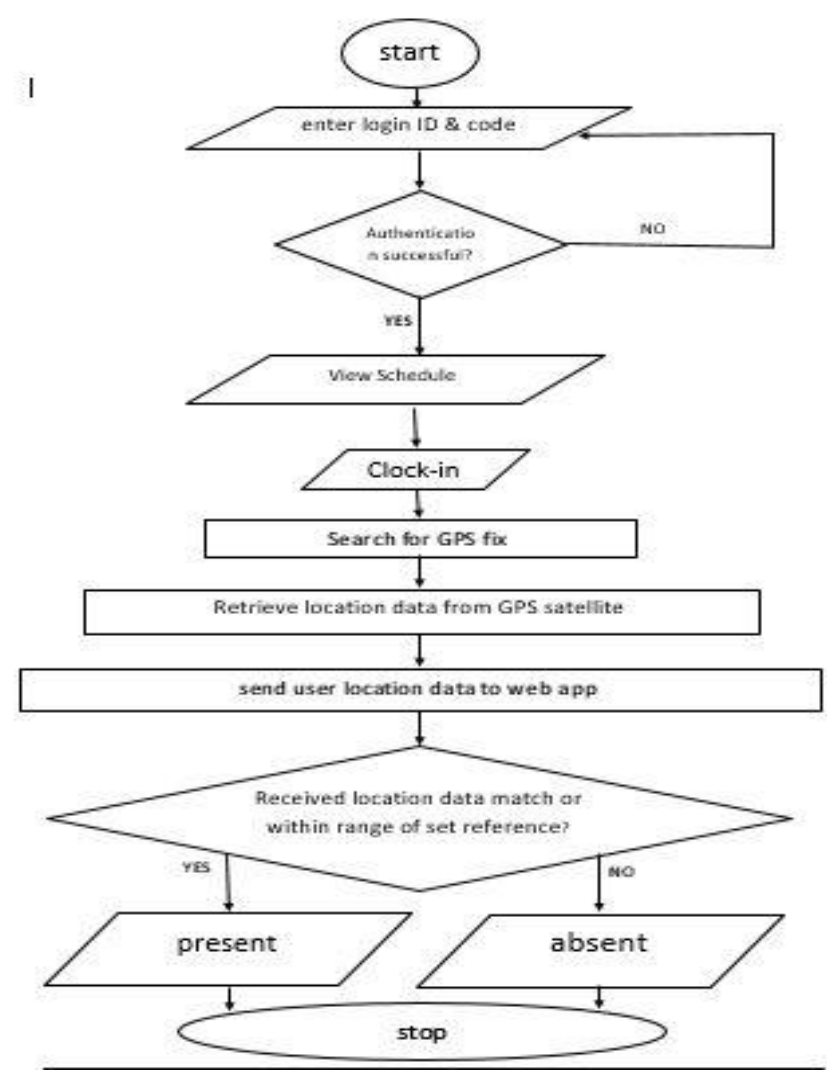

Fig. 4: Flowchart for the implementation of the proposed system

And then comparing this computed distance with a pre-set value called the tolerance. If the calculated distance is less than or equal to the tolerance, then the user is adjudged to be present. Otherwise elicits an adverse attendance judgment. The tolerance is a chosen radius around the designated venue, within which a positive attendance judgment can still be obtained.

There are some possible approaches to calculating geo-distance between points on the Earth surface given their latitudes and longitudes [12, 13]. Three common methods include: Haver-sine formula, spherical law of cosines, and Equi-rectangular approximation.

However, in this study, for the spherical law of cosines, the adopted formula for geodetic calculations allowing direct input of longitude and latitude values is $[13,16]$ :

$$
\begin{aligned}
\mathrm{d}=\operatorname{acos}[(\sin \omega 1)(\sin \omega 2) & \\
+ & (\cos \omega 1)(\cos \omega 2)(\cos \Delta \lambda) x R
\end{aligned}
$$

Here, $d=$ distance between scheduled lecture venue and the location of lecturer clock-in/clock-out (in kilometres); $\omega 1=$ Latitude of scheduled lecture venue (in radians); $\omega 2=$ Latitude of location of lecturer clock-in/clock-out (in radians); $\Delta \lambda=$ difference between longitude of scheduled lecture venue and longitude of location of lecturer clockin/clock-out (in radians); R, mean radius of earth $=$ $6371 \mathrm{~km}$.

It should be understood that whenever clock-in/clockout occurs in a location such that the value of $d$ in the equation above exceeds the set Tolerance range in the web App, the lecturer is adjudged to be absent. However, it should be noted that spherical trigonometry deals with spherical triangles on the surface of a sphere. In spherical trig., the role of straight lines is played by great circles.

\subsection{Hosting and Deployment}

The web application in this research work is hosted using the Heroku platform as a service. It appears as a subdomain under the Heroku main domain. Heroku and Nodejitsu are two common PaaS services for deploying node.js and MongoDB-based web applications although Heroku is more feature-rich than the latter $[14,15]$. Hosting on Heroku is achieved by using the 'Heroku create' command, and it requires first uploading the source code to the GitHub repository, as against using FTP as we see in traditional web hosting [15].

\subsection{System Interface}

\subsubsection{Home Page}

This is the first page of the web application, and its snapped shot is presented in Fig. 5. It displays the name of the application at the top and on the three tabs labeled Manage, Add Lecturer and Settings

\subsection{2 'Add Lecturer' Interface}

This is the interface where the administrator registers the course lecturers. It has the following fields: Lecturer ID, Lecturer Name and Code. The admin provides the course lecturers with their respective user ID and authorisation codes as these are the parameters needed for login and subsequent clocking on their android clients. After creating an account for the lecturer, then we can progress to the scheduling interface.

\subsection{3 'Class Scheduling' Interface}

Fig. 7 presents Class scheduling Interface. In this interface, the administrator inputs the details of the lectures to be anchored by the newly registered lecturer. Fields in this window include Day of The 
Week, Course, Course Code, Venue, Start Time, End Time, Longitude and Latitude. It is important to note that longitude and latitude values to be entered are those for the designated venue for the class being scheduled. The format should be in decimal and not in degrees and minutes. The location details of each venue are determined using appropriate tools or a readily available Android application like Latitude Longitude downloadable from Google Play Store. After this, click submit, and then done.

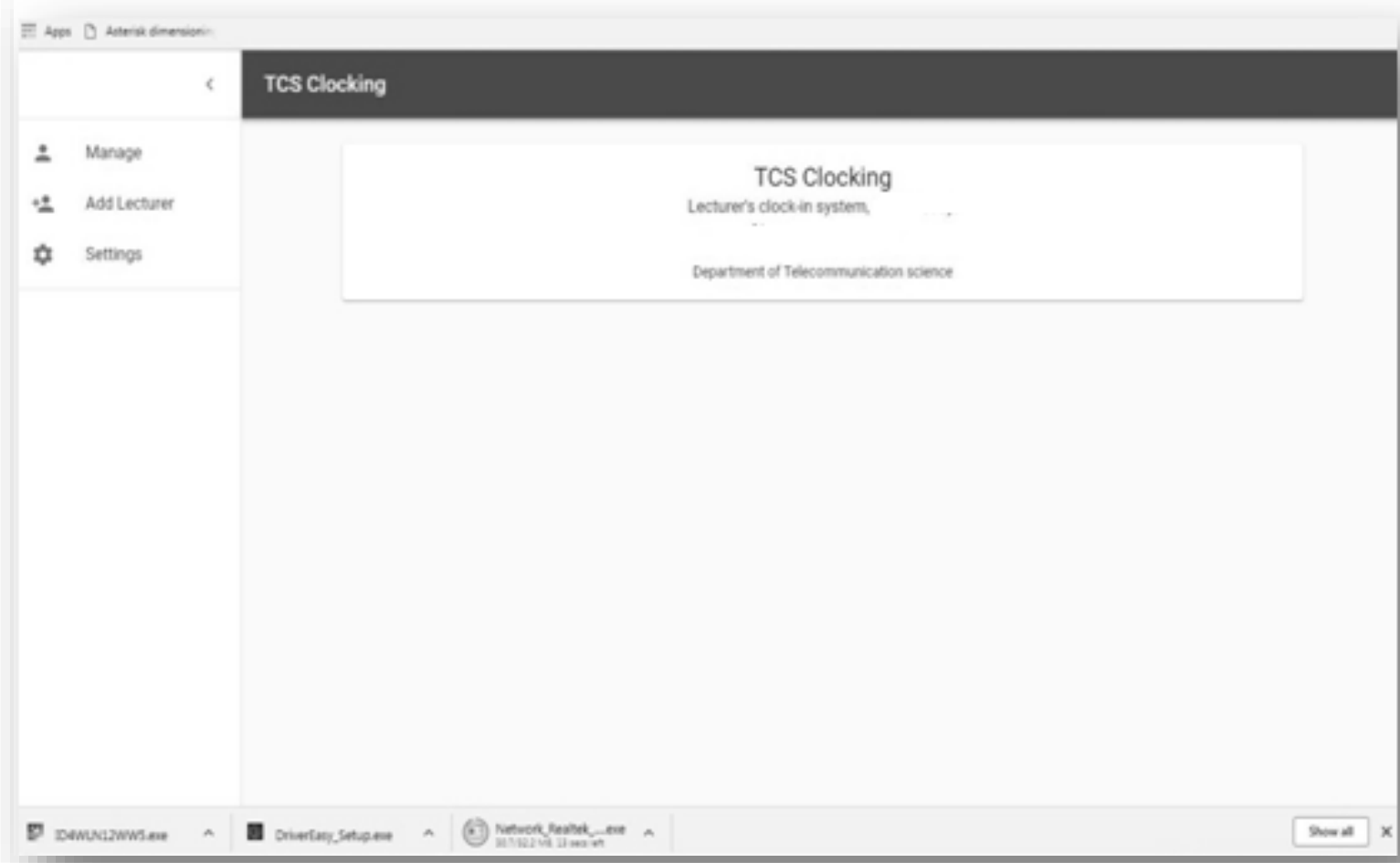

Fig. 5: Web application homepage

\section{TCS Clocking}

Enter Lecturer's Information

Authorisation Code"

Fig. 6: Web application 'Add lecturer' interface 
$\therefore$ Manage

+2 Add Lecturer

\$. Settings

\begin{tabular}{|c|c|c|}
\hline & \multicolumn{2}{|c|}{ Schedule Classes for Dr Olawoyin } \\
\hline Wednesday & & \\
\hline Couses. & counte coses. & venuer: \\
\hline Satellite Comm & TCS418 & London \\
\hline start tine & Eno Tinet & \\
\hline 03.00 PM & 05:00 PM & \\
\hline Lonatuce: & Lativuse: & \\
\hline 0.118092 & 51.509865 & \\
\hline
\end{tabular}

Fig. 7: Web application scheduling window

\subsection{Android Interfaces}

Figs. 8 and 9 present Android login and clock-in interfaces respectively. The android client functions mainly for clocking and sending the users' location details to the web application in the process; thus, it is a lightweight subsystem. Upon launching the application, it displays the name of the institution shortly before proceeding to the login screen with two fields for entering the 'User ID' and 'Access Code'.respectively. Next, it displays the scheduled lecture(s) associated with the entered ID, and to clockin, the user taps on the desired schedule. The Android client has the following interfaces: login interface, 'view schedule' interface and the clock-in interface.

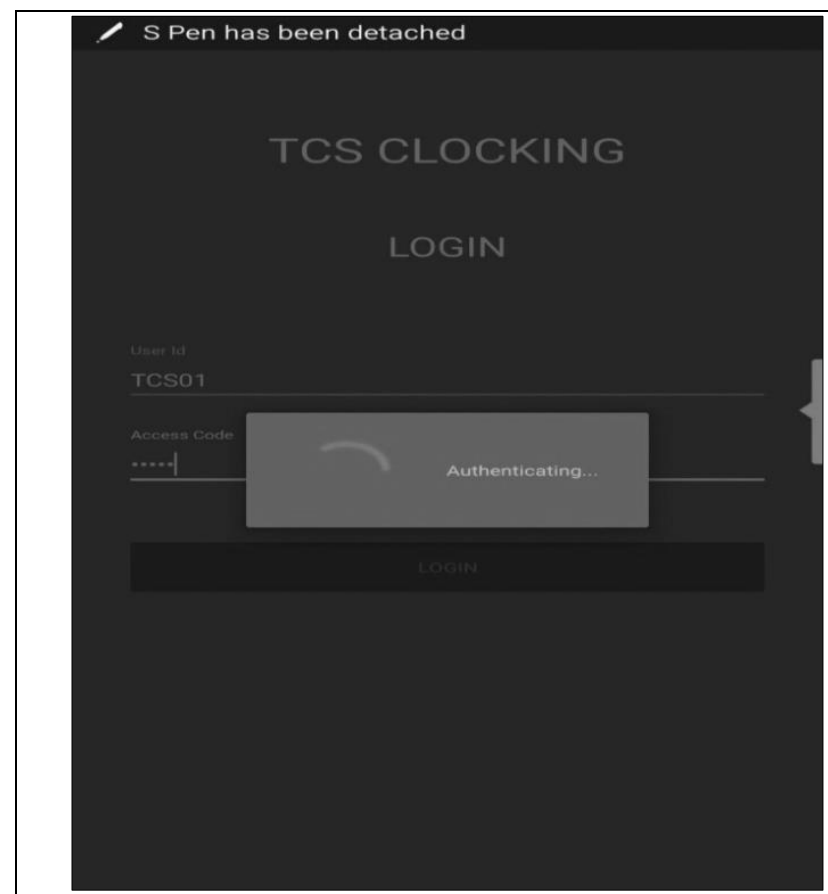

Fig. 8: Android login interface

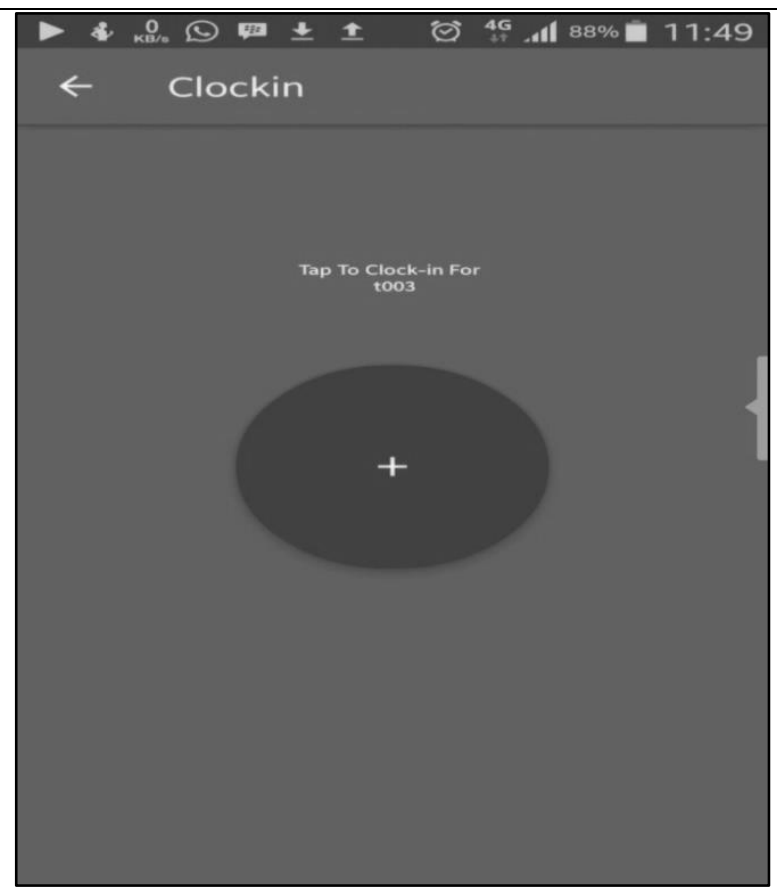

Fig. 9: Android clock-in interface 


\section{RESULTS AND OUTPUTS}

The web application and its associated functionalities/ privileges are reserved for the administration. The associated functionalities/privileges include: addition of users, scheduling classes for users, removing users, edit previously entered information, add a course for registered users, and view attendance.

In the case of the android client, after login, the user is only able to see the schedule(s) associated with the user ID logged in. Thus, the only two privileges for the android client user (lecturer) is to view applicable schedules and clock-in.

Fig. 10 shows the result of created accounts on the web app. From the above, clicking the view button displays all the scheduled classes for the lecturer concerned. To schedule additional classes for the lecturer under consideration, we click on the add course button.

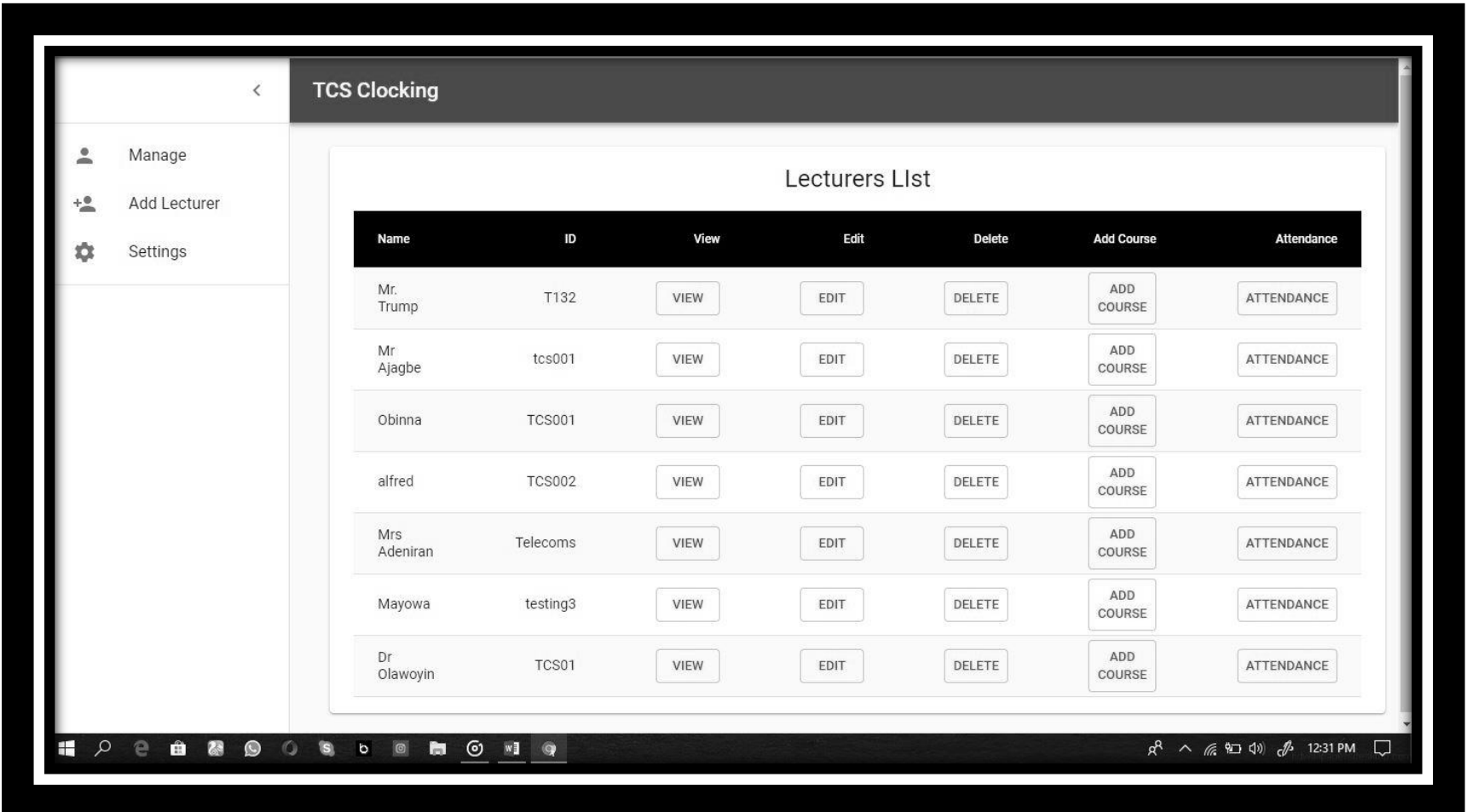

Fig. 10: List of registered users

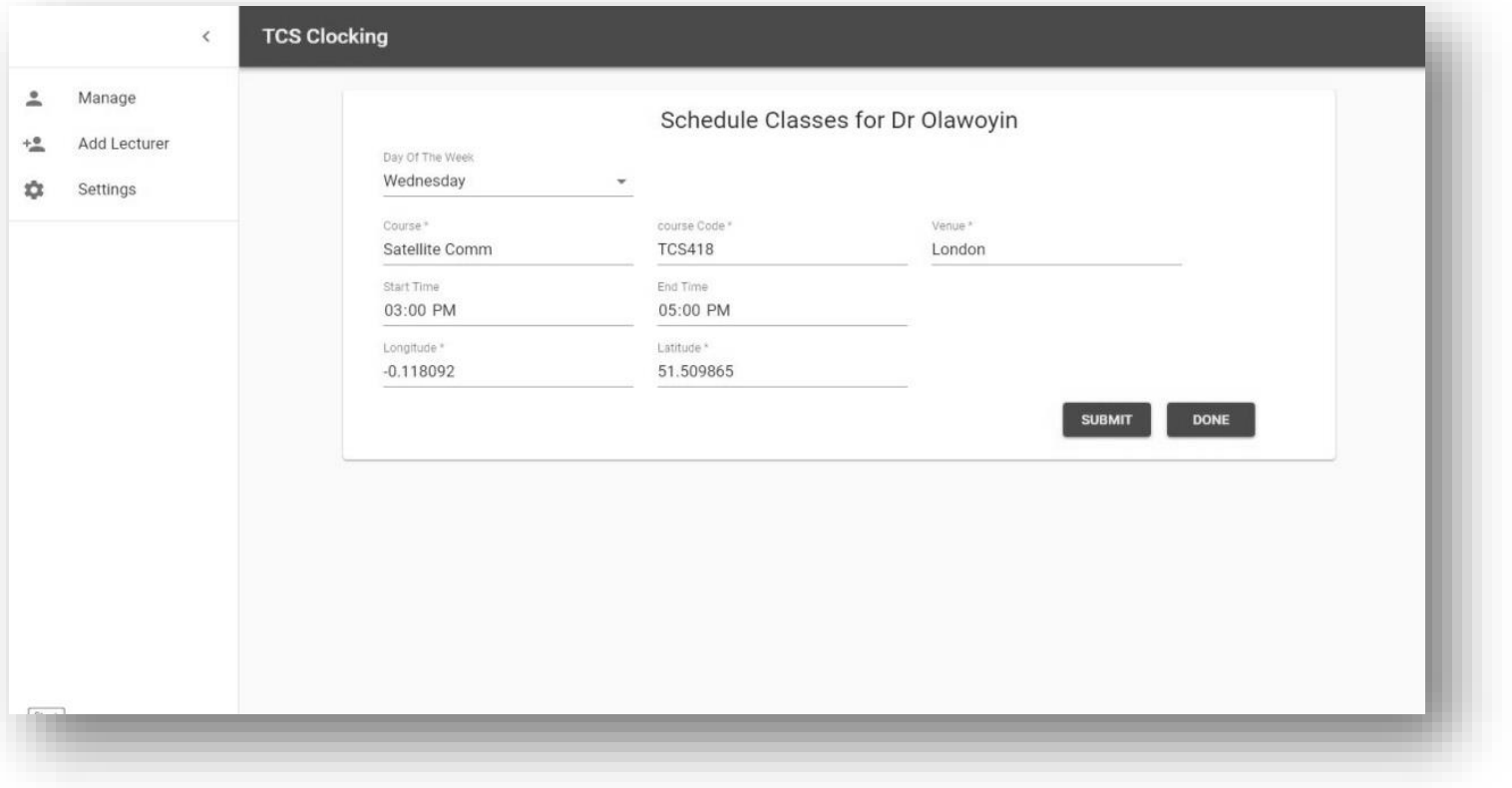

Fig. 11: scheduling window for Dr Olawoyin 
Fig. 11 shows the details as entered for the user $D r$ Olawoyin. The longitude and latitude fields are entered correctly to six decimal place. This view is available to the admin alone. It is important to note that the course name, course code and lecture venue corresponds with what is displayed on the mobile in Fig. 12. Fig. 12 shows that the class was scheduled for 3:00 pm to 5: $00 \mathrm{pm}$, the course is Satellite Comm, course code is TCS418 and venue in London.

In Fig. 13, the result shows when user Dr Olawoyin clocks in, on his mobile application. It is important to notice that the user clocks in at a time well within the stipulated schedule for the lecture which is $3: 00 \mathrm{pm}$ to 5:00 pm.

The above results are obtained by carrying out the following actions sequentially:

Step 1: The web administrator creates accounts for all the users;

Step 2: The administrator passes the different users' IDs and applicable authorisation codes to all the users.

Step 3: A user logs in on the mobile client; and on successful authentication, all classes scheduled for the user showing course, course code and venue are displayed;

Step 4: The user is required to ensure that locations are enabled in the Android system settings to allow the mobile to establish a GPS fix necessary for successful clocking;

Step 5: Usertaps on a schedule to activate the clocking interface for that schedule.

\section{CONCLUSION AND RECOMMENDATIONS}

Although various clocking solutions utilising other technologies exist, most require elaborate capital and operating costs. Ultimately, a GPS-based system is superior in many respects: net cost, ease of deployment and use, robustness, scalability, and minimal privacy concerns. The proposed clocking system is meant to drastically reduce the cost of clocking personnel, while at the same time, taking into consideration the peculiar nature of academic staff where flexibility is a necessary ingredient to maximise productivity. The system also, leverages on the staggering availability and reliability afforded by the U.S owned GPS. And because a very high percentage, if not all academic staff possess smart phones, deployment will be just as rapid as distributing the APK files and the users' IDs and authorisation codes. However, it should not be forgotten that this work is geared mainly towards concept validation and thus full-scale real-life implementation would do with a couple of improvements to notch up performance, such as the area of usability and security threats such as impersonation.

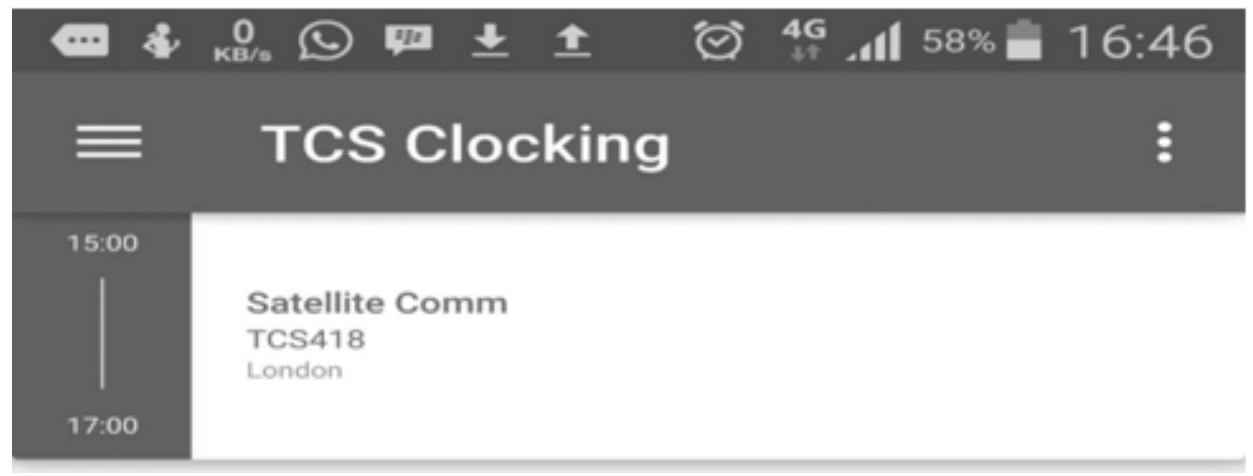

Fig. 12: Schedule as displayed on the user Dr Olawoyin's mobile.

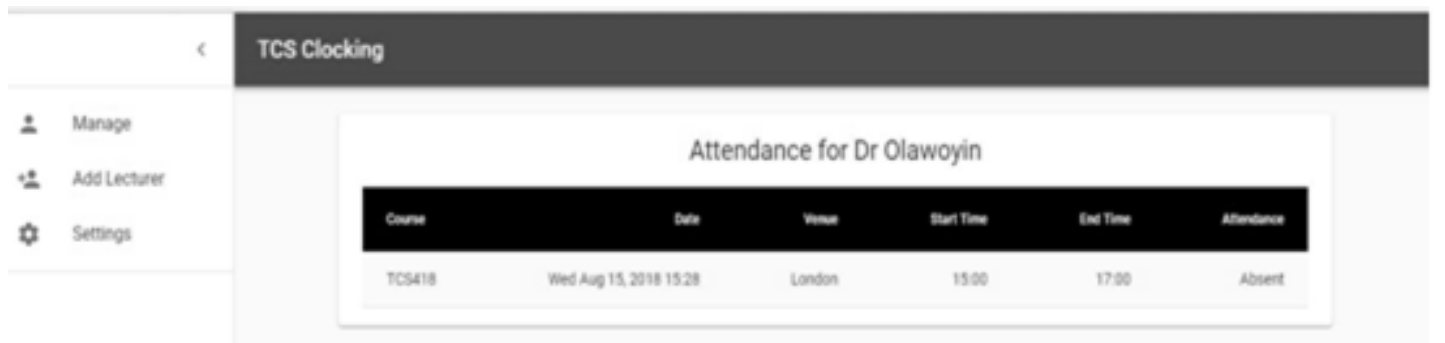

Fig. 13: Attendance window 
However, in the aspect of security, the possibility of cheating can hardly be totally eliminated in any system. All we can do is to implement a system that is highly robust and tedious to cheat. That said, it is recommended that we curb impersonation by noting the IMEI (International Mobile Equipment Identity) of each lecturer's mobile gadget and associate the same with the particular lecturer's logon details at the point of registration. This is to ensure that, whenever geolocation data is sent to the web app, the data is accompanied by the IMEI number of the sending client device at that point in time. Such that any discrepancies between this and the registered IMEI for the user account, triggers a negative attendance judgment. Secondly, the android client app can also be setup to always fetch the MSISDN (Mobile Subscriber Integrated Service Digital Network) number of the SIM in use in the device. And just like with the IMEI technique above, if an MSISDN other than the initially registered one is detected, clocking is also nullified. Thirdly, we can leverage on built-in fingerprint sensors in modern smart-phones to verify and authenticate users at the point of clocking. The above solutions are implementable on the program codes of the web and android applications.

\section{REFERENCES}

[1] Lende, L. and Patil, N.. "Location-Based Personal Task Reminder for Mobile Users in Wireless College Campus Environment(indoor and outdoor)", International Journal of Computing Techniques, Vol. 2, Number 1, 2015, pp 1-7.

[2] Masashi, N., Toshiyuki, T., Masashi, Y. "GPS Positioning Method under Condition of Only Three Acquired Satellites" The SICE Annual Conference 2008 the Univ. of ElectroCommunication (UEC), 2008, pp. 3487-3490.

[3] Cupido, U.O. "The Implementation of A Time and Attendance System at Stellenbosch Municipality A Change Management Perspective", University of Stellenbosch, 2011.

[4] Kim, Y., Walter, C.P., "Retrace and Disciplining Time Constant Effects on Holdover Clock Drifts in Chip-Scale Atomic Clock", Joint Conference of the European Frequency and Time Forum and IEEE International Frequency Control Symposium (EFTF/IFCS), Besancon, 2017, pp. 310-314.

[5] Umar, F., Muhammad, A., Hafiza, R.I., Oneeza, K., Sehrish, N., Asad, M.U., "Cost-effective wireless attendance and access control system", 2010, http://www.researchgate.net, 10.1109/ICCSIT.2010.5565099.

[6] Chiagozie, O.G. and Nwaji, O.G. "RFID-based attendance system with automatic door unit." Federal University of Technology Owerri. Academic Research International, vol. 2, Number 2, 2012, pp 168-183.

[7] Adeniran T. C. "Deployment of RFID-based card for University Staff and Students and its Significance on Privacy", International Journal of Information Processing and Communication, Vol. 3, Number (1\&2), 2015, pp 231-241.

[8] Uddin, M.S. Allayear, S.M., Das, N.C. and Talukder, F.A. "A Location-based time and attendance System," International Journal of Computer Theory and Engineering, Vol. 6, Number 1, 2014, pp 36-38.

[9] Agrawal, D.P., Bajaj, R. and Ranaweera, S.L. "GPS: Location tracking technology," Computer, vol. 35, Number 4, 2002, pp. 92-94.

[10] Chang, T.H., Wang, L.S., Chang, F.R. "A Solution to the III- Conditioned GPS Positioning Problem in an Urban Environment" IEEE Transactions on Intelligent Transportation Systems, Vol. 10, Number 1, 2009.

[11] Wang, E., Zhang, S. and Zhang, Z. "Research on the Incomplete Constellation GPS Positioning Algorithm by a Combined Altitude and Clock Bias Model," WASE International Conference on Information Engineering, Beidaihe, Hebei, 2010, pp. 219-222. doi: 10.1109/ICIE.2010.148.

[12] G.T. French. Understanding The GPS: An Introduction to The Global Positioning System. What It Is and How It Works. 1st edition, New York, GeoResearch Inc., 1996.

[13] Djuknic, G.M. and Richton, R.E. "Geolocation \& Assisted GPS", Computer, 2001, pp 123-125.

[14] Chodorow, K., "MongoDB: The Definitive Guide", Second edition, O'Reilly Media Inc., 2013.

[15] Satheesh, M., Krol, J. "Web Development with MongoDB and NodeJS", Second edition, Packt Publishing, 2015.

[16] Movable Type Scripts, "Calculate distance, bearing and more between Latitude/ Longitude points", Retrieved $6^{\text {th }}$ March, 2018, from: http://www.movable-type.co.uk/scripts/l atlong.html. 